\title{
Study on Reliability Analysis for Braking System Parts Based on Hybrid Censoring Test under Small Sample Size
}

\author{
Jianwei Yang ${ }^{1,2, *}$, Jinhai Wang ${ }^{1,2}$, Chunqing Zhao ${ }^{1,2}$ and Fumin Wang ${ }^{3}$
}

\author{
${ }^{I}$ School of Machine-electricity and Automobile Engineering, Beijing University of Civil Engineering Architecture. Bei- \\ jing, 100044, China; ${ }^{2}$ Beijing Key Laboratory of Performance Guarantee on Urban Rail Transit Vehicles, Beijing Uni- \\ versity of Civil Engineering Architecture, Beijing, 100044, China; ${ }^{3}$ School of Mechanical Engineering, Taiyuan Univer- \\ sity of Science and Technology, Taiyuan, Shanxi, 030024, China
}

\begin{abstract}
In order to further reduce time and resource consuming and describe hazard curve of parts in bathtub shape, the likelihood function of Modified Weibull distribution based on hybrid censoring test is derived. Meanwhile, for improving reliability analysis accuracy under small sample size, Bayesian method using Gamma distribution and Uniform distribution as the prior distribution is applied to analyze reliability with hybrid Markov chain Monte Carlo algorithm. And then, taking a valve in the barking system of railway vehicle as an example, the scheme of hybrid censoring test is made. Computing result shows that the method can improve the problem of reliability analysis under small sample size.
\end{abstract}

Keywords: Bayesian method, hybrid censoring test, hybrid markov chain monte carlo algorithm, modified weibull distribution, small sample size.

\section{INTRODUCTION}

The reliability of rail vehicle brake parts will directly affect the reliability of rail vehicle braking system, which will affect the safety of the vehicle. According to the statistical analysis data, the braking faults of metro rail transit vehicle are mainly focused on the air compressor, electronic brake unit, brake control module and the emergency brake, brake shoe, park brake valve (pkBV) and other components [1]. Generally speaking, the reliability estimation of the braking system cannot only start from the system, but also the parts, or both system and the important parts are balance the simultaneously. Traditionally, the reliability assessment is to make statistics analysis on the lifetime data for a large amount of test samples (usually $n>30$ ), which means that the reliability test need to spend a lot of time and fund. Meanwhile, consumption of rail vehicle will be greater on account of the longer design life, more expensive test specimen, more parts and more complex system. Therefore, a kind of method is needed to make a reliability estimation for the life test in the allotted amount of time under a small sample.

\section{THE BAYESIAN METHOD PROPOSED}

It is not designed and manufactured in a completely unprecedented condition for the a majority of parts. Generally, they are either based on the improved design of some old parts or mature components which is similar to be used. or there are similar parts manufactured in the same line or the experience of manufacturing similar production, or there is the lifetime data of test parts in different environments or the simulation data calculated, in the computer, which is referred to as prior data. In the problems of traditional reliability assessment, the prior data tend to be ignored. And in order to obtain estimation results with higher reliability, it needs to get a large number of lifetime data in the current environment. Therefore, the tradional reliability assessment methods become stretched under small sample size.

In order to solve the problem of small sample, the Bayes method has been widely used [2-6]. Bayesian school thinks the parameter as a kind of random variable, obeying a certain statistical law in statistic, which shows that through the probability distribution it can express the distribution of parameter, and each specific sample distribution as just an implementation of the paramerters in the distribution. Therefore the prior information and sample information can be combined using the Bayes formula, and by method of borrowing prior information, the accuracy of reliability assessment under small sample size condition can be ultimately improved according to the current sample information to modify. For the purpose of solving parameter estimation through subsequently using Markov chain Monte Carlo algorithm, the simplified Bayes formula can be expressed as follows:

$h(\theta \mid x)=L \cdot(x \mid \theta) \times \pi(\theta)$

where, $\mathrm{h}(\theta \mid \mathrm{x})$ means the posterior distribution which is the ultimate goal of statistical inference; $\mathrm{L}(\mathrm{x} \mid \theta)$ is the likelihood function containing all information of the current test samples about the parameter $\theta ; \pi(\theta)$ means prior distribution which contains all the priori information. It can be made out that the posteriori estimation of the parameters is the result of the intersection of the prior information and the sample information through the Bayes method from the type (1). 


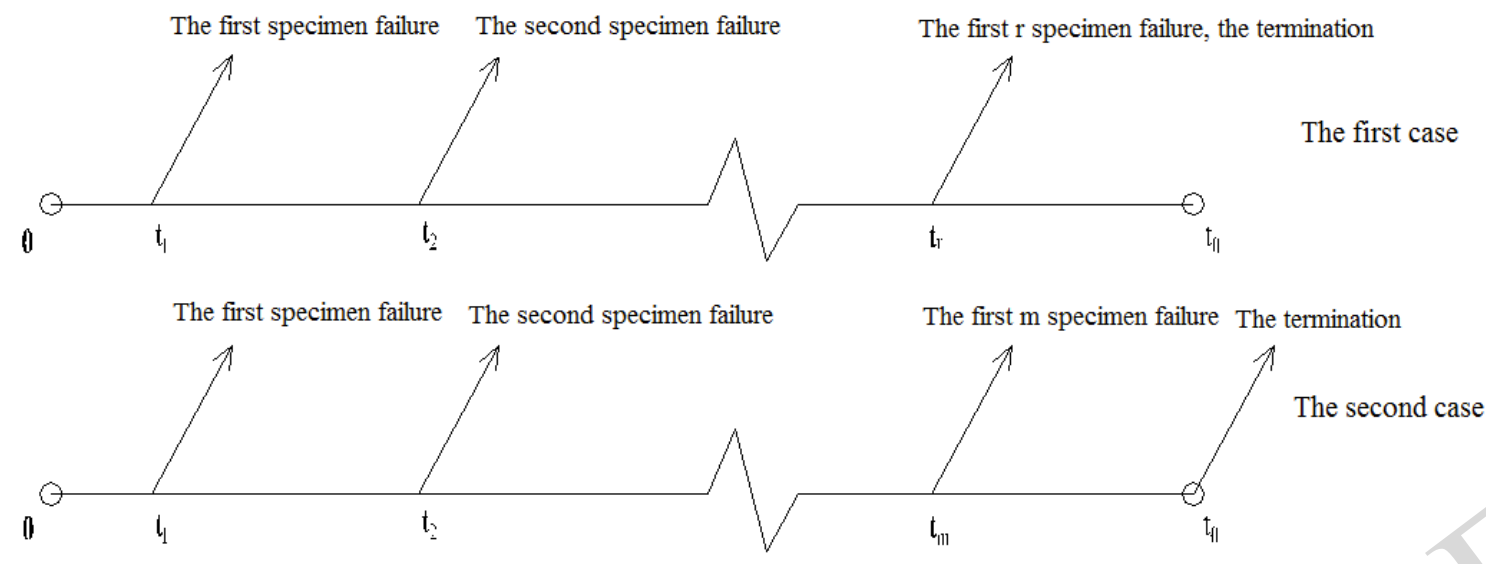

Fig. (1). The time process of hybrid censoring lifetime test.

\section{RELIABILITY ASSESSMENT MODEL}

\subsection{Modified Weibull Distribution and the Likelihood Function under Hybrid Censoring Lifetime Test}

Modified Weibull distribution can describe the bathtub curve of life failure rate for the parts [7]. The cumulative failure probability function, reliability function, failure probability density function and failure rate function can be given as follows:

$$
\begin{aligned}
& F(t)=1-\exp \left[-a \cdot t^{b} \cdot \exp (\lambda \cdot t)\right\rfloor \\
& R(t)=\exp \left\lfloor a \cdot t^{b} \cdot \exp (\lambda \cdot t)\right\rfloor \\
& f(t)=a \cdot(b+\lambda \cdot t) \cdot t^{b-1} \cdot \exp (\lambda \cdot t) \cdot \exp \left[a \cdot t^{b} \cdot \exp (\lambda \cdot t)\right\rfloor \\
& h(t)=a \cdot(b+\lambda \cdot t) \cdot t^{b-1} \cdot \exp (\lambda \cdot t)
\end{aligned}
$$

The shape of failure rate function $\mathrm{h}(\mathrm{t})$ only depends on the value of parameter $b$, as the specific condition described in the following sections: when $\mathrm{b} \geq 1, \mathrm{~h}(\mathrm{t})$ is monotonously increasing, which can describe the wear-out failure period of parts; when $0<b<1, h(t)$ will first decrease as time $t$ increases, and then increase, which shows the shape of bathtub curve to describe the reliability of the components in the whole life cycle. Particularly, Modified Weibull distribution will degrade into two-parameter Weibull distribution when $\lambda=0$; Modified Weibull distribution will degrade into Log-Gamma distribution when $b=0$.

Censoring test is a common measurement means to shorten the test cycle and test money in survival analysis. There are four kinds of common lifetime test: time truncation censoring lifetime test (Type I censoring test), number truncation censoring lifetime test (Type II censoring test), hybrid censoring test and update type censoring lifetime test. Many researchers at home and abroad have made a lot of research on the censoring test of Modified Weibull distribution [8-10]. This paper will make modeling and analysis of hybrid censoring lifetime test.

Hybrid censoring lifetime test is the synthesis of time truncation censoring lifetime test and number truncation censoring lifetime test for the general test method as follows: randomly select $\mathrm{n}$ test specimens from an overall samples for lifetime test, and in the meanwhile, give a positive number t0 and positive integer $r(r \leq n)$.
(1) If lifetime data of $r$ tests can be observed before the time T0, the deadline $\zeta$ equals to $t_{r}$, which belongs to number truncation censoring lifetime test;

(2) If the time to is reached before getting lifetime data $r$ tests, the deadline $\zeta$ equals to $t_{0}$, which belongs to time truncation censoring lifetime test.

The exact time process is shown in Fig. (1).

For the hybrid censoring lifetime test, the likelihood function can be expressed as follows:

$$
L(x \mid \theta)=C_{n}^{m} \prod_{i=1}^{m} f\left(x_{i}\right)[1-F(\zeta)]^{R}
$$

where, $\mathrm{m}$ is the number of the destructive test specimens before the termination of hybrid censoring lifetime test, and $\mathrm{m} \leq \mathrm{r}$; $\mathrm{R}$ is the number of unbroken test specimens, that is, $\mathrm{R}=\mathrm{n}-\mathrm{m}$.

Through putting Eq. (2) and (4) into Eq.(6), the likelihood function of hybrid censoring lifetime test of Modified Weibull distribution can be obtained as follows:

$$
\begin{aligned}
& L(x \mid a, b, \lambda)=C_{n}^{m} a^{m}\left[\prod_{i=1}^{m}(b+\lambda \cdot t) \cdot t^{b-1}\right] \exp \\
& ,\left[\lambda \sum_{i=1}^{m} t_{i}-a\left(\sum_{i=1}^{m} t_{i}^{b} e^{\lambda t_{i}}+R \xi^{b} e^{\lambda \xi}\right)\right]
\end{aligned}
$$

\subsection{Bayesian Inference}

In the assumption process of prior distribution, statisticians will usually suppose that the parameter which is from 0 to infinity obeys Gamma distribution or Inverse-Gamma distribution on account of the same number field. And for parameter with the number field on a closed interval, it usually uses the uniform distribution as the prior distribution according to the principle of indifference. Although the principle of indifference can in logically explain the reason for using the uniform distribution assumption in the insufficient prior information condition, there are still many statisticians to have proposed many paradoxes of this assumption for a long time [11-13]. So when it sets the prior distribution, uniform distribution should be used as little as possible as prior distribution. For the three-parameter Modified Weibull dis- 
tribution, it is assumed that they obey the prior distribution as follows:

$$
\begin{aligned}
& \pi(a) \propto a^{\gamma_{a}-1} \exp \left(-\eta_{a} a\right) \\
& \pi(b) \propto b^{\gamma_{b}-1} \exp \left(-\eta_{b} b\right) \\
& \pi(\lambda) \propto \frac{1}{M}
\end{aligned}
$$

Put Eq. (7)- (10) into Eq.(1), which can obtain joint posterior probability density function as follows:

$$
\begin{aligned}
h(a, b, \lambda \mid x) & \propto a^{m+\gamma_{a}-1} b^{\gamma_{b}-1}\left[\prod_{i=1}^{m}(b+\lambda \cdot t) \cdot t^{b-1}\right] \\
& \exp \left[\lambda \sum_{i=1}^{m} t_{i}-a\left(\sum_{i=1}^{m} t_{i}^{b} e^{\lambda t_{i}}+R \xi^{b} e^{\lambda \xi}\right)-\eta_{a} a-\eta_{b} b\right]
\end{aligned}
$$

Through regularization of integral content, the distribution function of joint posterior probability density function can be received. And then joint posterior probability density function of each parameter can be obtained after integrating each integral. However, the integral is often very difficult because of the complex model which is unable to get the form of analytical solution, so it mostly uses a numerical method to solve the value in this case. On the other hand, the calculated amount of numerical method is enormous for high dimensional model with the multiparameter, and in order to solve this problem, Monte Carlo algorithm can be adopted to calculate the value of the integral.

\section{HYBRID MARKOV CHAIN MONTE CARLO AL- GORITHM}

The basic idea of Markov chain Monte Carlo algorithm matters what follows, starting from arbitrary initial value, takes a sample in the proposal distribution, and accepts the sample with acceptance probability $\alpha$ establishing a Markov chain which regards the stationary distribution as target distribution. When the sampling results are more enough, it can be thought that the sampling result converges to the stationary distribution through burn-in a part of samples in front. Finally, it has statistical inference for the samples obeying posterior distribution to get the estimation of relevant parameters. According to Eq. (11), kernel function of posterior distribution for each parameter can be obtained by keeping the other parameters constant, and the kernel of objective function is as follows:

$$
\begin{gathered}
h(a \mid x, b, \lambda) \propto a^{m+\gamma_{a}-1} \exp \left[-a\left(\sum_{i=1}^{m} t_{i}^{b} e^{\lambda t_{i}}+R \xi^{b} e^{\lambda \xi}-\eta_{a}\right)\right] \\
h(b \mid x, a, \lambda) \propto b^{\gamma_{b}-1}\left[\prod_{i=1}^{m}(b+\lambda \cdot t) \cdot t^{b-1}\right] \\
\exp \left[a\left(\sum_{i=1}^{m} t_{i}^{b} e^{\lambda t_{i}}+R \xi^{b} e^{\lambda \xi}\right)-\eta_{b} b\right] \\
h(\lambda \mid x, a, b) \propto\left[\prod_{i=1}^{m}(b+\lambda \cdot t) \cdot t^{b-1}\right] \exp \left[\lambda \sum_{i=1}^{m} t_{i}-a\left(\sum_{i=1}^{m} t_{i}^{b} e^{\lambda t_{i}}+R \xi^{b} e^{\lambda \xi}\right)\right]
\end{gathered}
$$

There are two kinds of ordinary algorithms including Metropolis algorithm and Gibbs algorithm for sampling.
Metropolis algorithm can carry out sampling on all objective distribution, but the efficiency of sampling cannot be guaranteed. While the sampling efficiency of Gibbs algorithm is very high with the disadvantage of sampling frame which only includes the generic distribution. According to Eq.(12), posterior distribution $\mathrm{h}(\mathrm{a} \mid \mathrm{x}, \mathrm{b}, \lambda)$ is the kernel of Gamma distribution with parameter $\left(\gamma_{a}+m, \sum_{i=1}^{m} t_{i}^{b} e^{\lambda_{i}}+R \xi^{b} e^{\lambda \xi}-\eta_{a}\right)$, so Eq.(12) is known as conditional posterior probability density function with parameter a. Eq. (13) and (14) are not the kernel of common distribution, so they are marginal posterior probability density of the parameter $b$ and $\lambda$. Therefore, for $\mathrm{h}(\mathrm{a} \mid \mathrm{x}, \mathrm{b}, \lambda)$, Gibbs algorithm can be used for sampling; for $\mathrm{h}(\mathrm{b} \mid \mathrm{x}, \mathrm{a}, \lambda)$ and $\mathrm{h}(\lambda \mid \mathrm{x}, \mathrm{a}, \mathrm{b})$, Metropolis algorithm is used to carry out sampling. The sample received through sampling the kernel function using hybrid Markov chain algorithm is the statistical sample obeying posteriori distribution. The concrete steps of the algorithm are as follows:

(1) Generate the initial value of Markov chain including $a(0), b(0)$ and $\lambda(0)$ to receive $T_{\max }$ and $T_{\text {burn-in }}$, and suppose that $T=0$;

(2) $T=T+1$;

(3) From Gamma $\left(\gamma_{a}+m, \sum_{i=1}^{m} t_{i}^{b} e^{\lambda t_{i}}+R \xi^{b} e^{\lambda \xi}-\eta_{a}\right)$, generate a new value $a(T)$;

(4) From $\mathrm{U}\left(0, M_{b}\right)$, generate a candidate point $b^{*}$;

(5) From the $\mathrm{U}(0,1)$, generate a random number $u_{b}$;

(6) From $\alpha_{b}=\min \left\{1, \frac{\pi\left(b^{*} \mid x, a(T), \lambda(T-1)\right)}{\pi(b(T-1) \mid x, a(T), \lambda(T-1))}\right\}$, calculate the acceptance rate;

(7) If $\mathrm{u}_{\mathrm{b}} \leq \alpha_{b}$, then $b(T)=b^{*}$; or $b(T)=b(T-1)$;

(8) From $\mathrm{U}\left(0, M_{\lambda}\right)$, generate a candidate point $\lambda^{*}$;

(9) From the U(0,1), generate a random number $u_{\lambda}$;

(10) From $\alpha_{\lambda}=\min \left\{1, \frac{\pi\left(\lambda^{*} \mid x, a(T), \mathrm{b}(T)\right)}{\pi(\lambda(T-1) \mid x, a(T), \mathrm{b}(T))}\right\}$, calculate the acceptance rate

(11) If $u_{\lambda} \leq \alpha_{\lambda}$, then $\lambda(T)=\lambda^{*}$; or $\lambda(T)=\lambda(T-1)$;

(12) Repeat step (2) to (11) until $T=T_{\max }$;

(13) Using Monte Carlo algorithm, calculate estimators of parameters after burn-in period, respectively.

where, $M_{b}$ is the sample range of parameter $b ; M_{\lambda}$ is the sample range of parameter $\lambda ; \mathrm{T}_{\max }$ is the length of the Markov chain; $\mathrm{T}_{\text {burn-in }}$ is the length of burn-in.

\section{EXAMPLE}

Rail vehicle braking system is connected by many valves which are easier to have the wearing breakdown due to the constant opening and closing, so it needs to assess the reliability. There is a group of lifetime test data of a valve with 
Table 1. The hybrid censoring lifetime test data of a valve.

\begin{tabular}{|c|c|c|c|c|c|}
\hline$i$ & 1 & 2 & 3 & 4 & 1.92 \\
\hline $\mathrm{t}_{i}(1000 \mathrm{~h})$ & 0.67 & 1.79 & 1.88 & Failure data & Censoring Data \\
\hline & Failure data & Failure data & Failure data & 9 & 10 \\
\hline$i$ & 6 & 7 & 8 & 2.00 & 2.00 \\
\hline $\mathrm{t}_{i}(1000 \mathrm{~h})$ & 2.00 & 2.00 & 2.00 & Censoring Data & Censoring Data \\
\hline
\end{tabular}
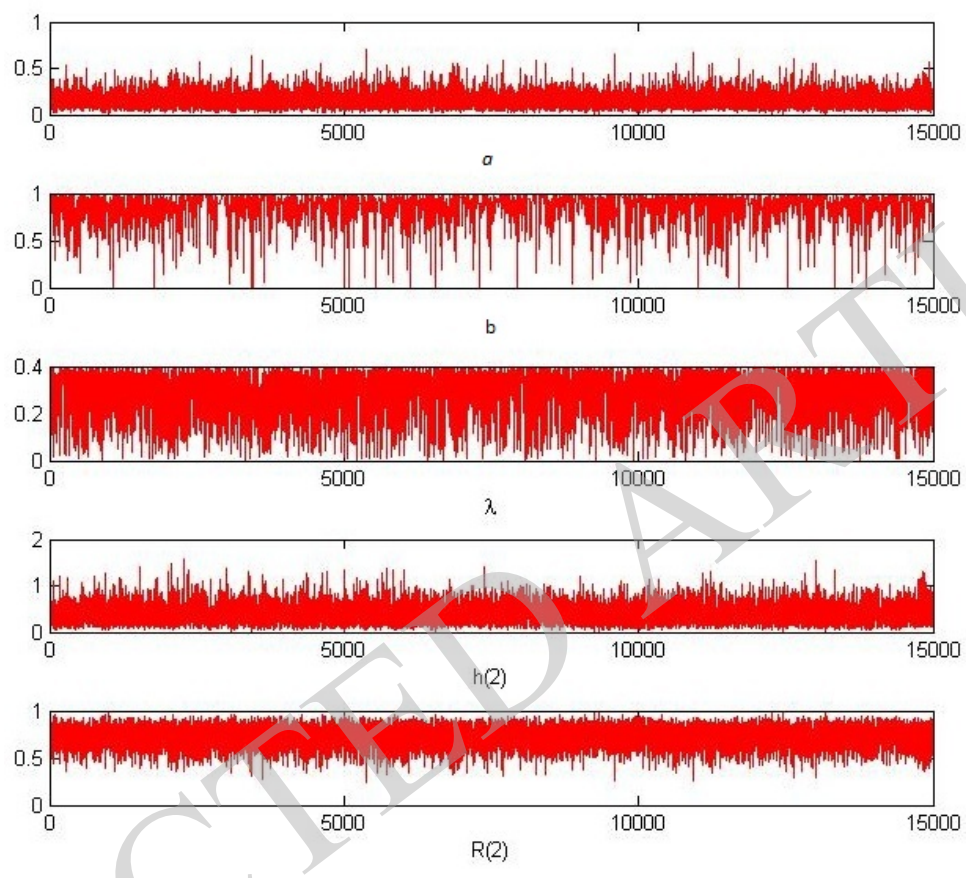

Fig. (2). Markov chain of parameter $a, b, \lambda, R(2)$ and $h(2)$.

the number of samples $n=10$, and the scheme design of hybrid censoring lifetime test stops when $\mathrm{t}_{0}=2$ or $\mathrm{r}=6$ to shorten the test time or reduce the number of loss specimen. Reliability evaluation demands to solve the reliability $\mathrm{R}(2)$ and failure rate $h(2)$ after $2000 \mathrm{~h}$ according to each estimated parameter. On the basis of test, it has reached time $t_{0}$ when $\mathrm{m}=5$, and the final test data is as shown in Table $\mathbf{1}$.

According to prior information, the estimation of hyperparameter for prior distribution can be obtained using moment estimation, least squares regression, empirical Bayes method, etc. In the example, hyper-parameter of prior distribution is determined by expert experience, and for parameter a, there is not prior information to be borrowed so that this paper supposes hyper-parameter $\gamma_{\mathrm{a}}=0$ and $\eta_{\mathrm{a}}=0$. For parameter $b$, there are some early failure test specimens which are only a small amount of specimens on account of the reliability data in the past. The average of $b$ given by expert experience is 0.6 , and variance is 0.1 . Meanwhile the hyperparameter $\gamma_{\mathrm{a}}=0$ and $\eta_{\mathrm{a}}=0$ can be obtained by using moment estimation method. Then for $\lambda$, take its hyper-parameter $\mathrm{M}=0.4$. In addition, using software $\mathrm{R}$ this paper programmes for the method given in Section 3, and makes parameter Bayes estimation of the experimental data above. Iterative process of the Markov chain for each parameter is shown in Fig. (2).

The histogram of each parameter is shown in Fig. (3) after the burn-in period.

Through Monte Carlo algorithm, it can obtain the estimation of each parameter including, $\hat{a}=0.15, \hat{b}=0.82$ and $\hat{b}=0.28$. Meanwhile, the estimation of reliability and failure rate after $2000 \mathrm{~h}$ can also be received which are $\hat{R}(2)=75.33 \%$ and $\hat{h}(2)=0.31$. According to the results received, the curve comparison of prior distribution and posterior distribution about $b$ is drawn as showed in Fig. (4).

Finally, it can get the failure rate curve according to the three parameters value, as showed in Fig. (5).

\section{CONCLUSION}

(1) Under the fusion of the Bayes method, the probability density curve of posterior distribution $\lambda$ is modified by prior information from Fig. (4) so that the accuracy of reliability evaluation is improved under small sample size condition. 

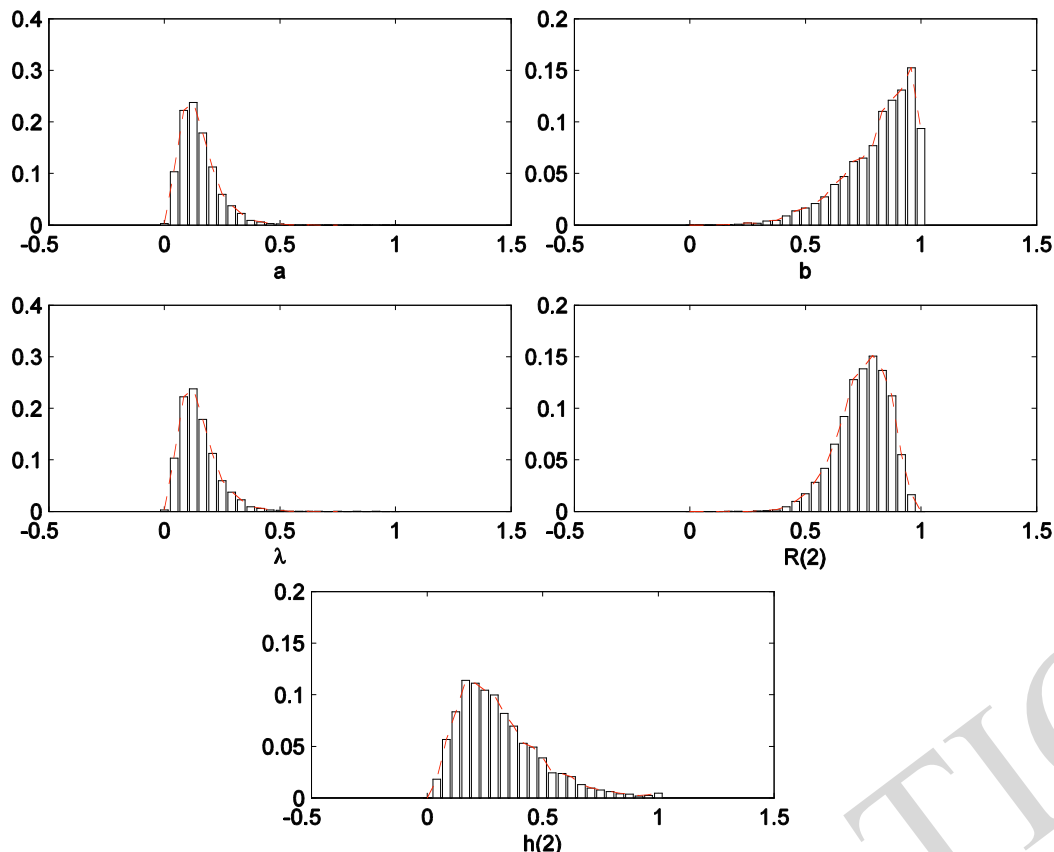

Fig. (3). The sampling histogram of each parameter $a, b, \lambda, R(2)$ and $h(2)$.

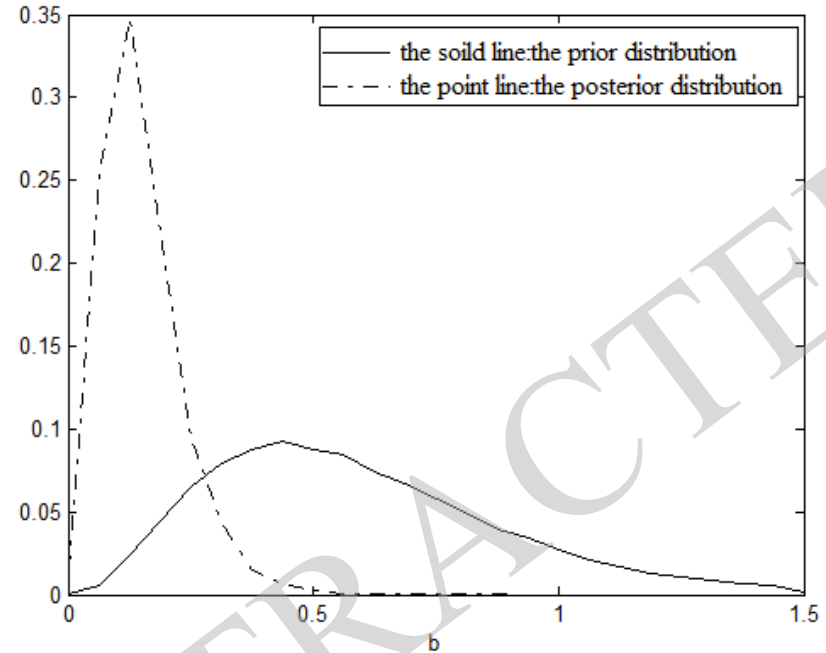

Fig. (4). The comparison of prior distribution and posterior distribution about b.

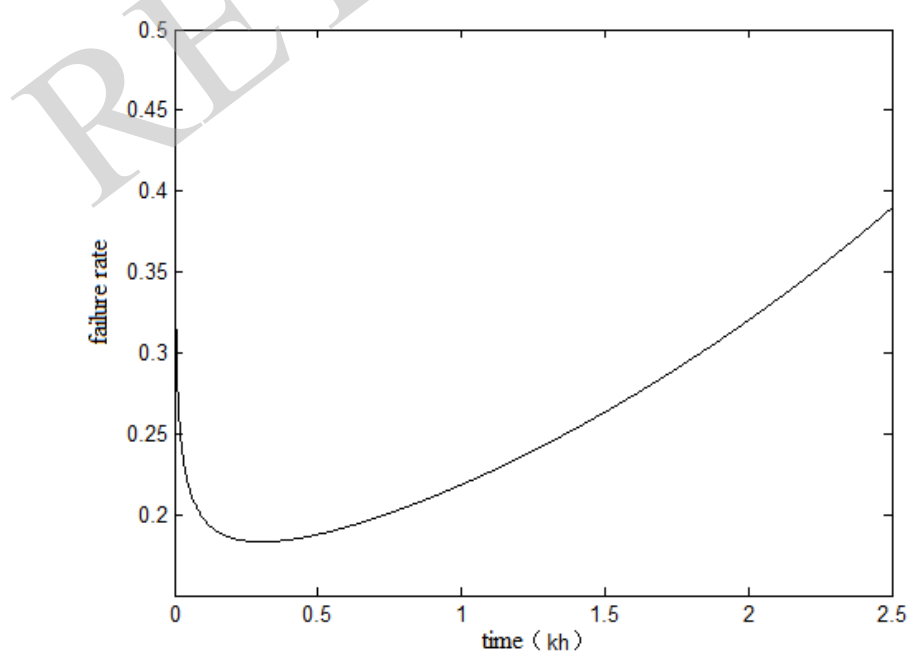

Fig. (5). The failure rate of a valve.
Meanwhile, combing with the advantage of hybrid censoring lifetime test, the reliability evaluation of braking valve can be completed in a shorter time and less resource condition.

(2) It is clear from Fig. (2) that parameters $b$ and $\lambda$ have great runout in the iterative process of Markov chain, which indicates that the alternative points by $b$ and $\lambda$ have higher acceptance rate, and then through matching up highefficiency Gibbs sampling, the efficiency of hybrid Markov chain Monte Carlo algorithm is proved.

(3) It is clear from Fig. (5) that Modified Weibull distribution can be used to describe bathtub curve of failure rate for valve which is consistent with the real situation provided by life data, using which reliability prediction of other parts for the whole life cycle can be carried out.

\section{CONFLICT OF INTEREST}

The authors confirm that this article content has no conflict of interest.

\section{ACKNOWLEDGEMENTS}

This paper was supported by the national natural science fund project (51175028), Beijing outstanding talent training projects (2010D005017000007), the International Science \& Technology Cooperation Program of China (2014DFR 70280) and BUCEA Urban Rural Construction and Management Industry Research Development Collabration Post Graduate Training Centre.

\section{REFERENCES}

[1] J. Cheng, and H. Li, "Reliability analysis of rolling-stock brake system for Shanghai lineal extension," Mechanical Research and Application, vol. 5, pp. 45-46. 2009.

[2] J. Dong, Study on Urban Rail Vehicle Brake System Reliability Modeling and Simulation, Shanxi: Taiyuan University of Science and Technology, 2012. 
[3] M. Guida, and G. Pulcini, "Automotive reliability inference based on past data and technical knowledge," Reliability Engineering \& System Safety, vol. 76, pp. 129-137, 2002.

[4] D. Lord, and L. F. Miranda-Moreno, "Effects of low sample mean values and small sample size on the estimation of the fixed dispersion parameter of Poisson-gamma models for modeling motor vehicle crashes: A Bayesian perspective," Safety Science, vol. 46, pp. 751-770, 2008.

[5] C. Hong, S. Zhang, and J. Zhang, The Analysis and Evaluation of Bayes, National University of Defense Technology Publishing, Changsha, 2004.

[6] J. Guang. "Performance reliability modeling and estimation for space bearing under small sample circumstance," Journal of $\mathrm{Na}$ tional University of Defense Technology, vol. 32, pp. 133-137, 2010.

[7] C. D. Lai, and M. Xie, "A modified weibull distribution," IEEE Transactions on Reliability, vol. 52, pp. 33-37, 2003.

[8] S. K. Upadhyay, and A. Gupta, "A Bayes analysis of modified Weibull distribution via Markov chain Monte Carlo simulation,"
Journal of Statistical Computation and Simulation, vol. 80, pp. 241-254, 2010.

[9] H. K. T. Ng, "Parameter estimation for a modified weibull distribution, for progressively type-II censored samples," IEEE Transactions on Reliability, vol. 54, pp. 374-380, 2005.

[10] A. A. Soliman, A. H. Abd-Ellah, N. A. Abou-Elheggag, and E. A. Ahmed, "Modified Weibull model: A Bayes study using MCMC approach based on progressive censoring data," Reliability Engineering \& System Safety, vol. 100, pp. 48-57, 2012.

[11] Gillies D, Philosophical Theories of Probability, Routledge, London: 2000.

[12] N. Metropolis, A. W. Rosenbluth, M. N. Rosenbluth, A. H. Teller, and E. Teller "Equation of state calculations by fast computing machines," The Journal of Chemical Physics, vol. 21, pp. 1087-1092, 1953.

[13] S. Geman, and D. Geman, "Stochastic relaxation, gibbs distributions, and the bayesian restoration of images," IEEE Transactions on Pattern Analysis and Machine Intelligence, vol. PAMI-6, no. 6, pp. 721-741, 1984.

(C) Yang et al.; Licensee Bentham Open.

This is an open access article licensed under the terms of the Creative Commons Attribution Non-Commercial License (http://creativecommons.org/licenses/by-nc/3.0/) which permits unrestricted, non-commercial use, distribution and reproduction in any medium, provided the work is properly cited. 